\title{
Identification of loci determining resistance of spring barley to spot and net blotch, using association mapping approach
}

\author{
I.V. Bykova ${ }^{1 *}$, S.A. Gorobets ${ }^{1}$, N.M. Lashina ${ }^{2}$, V.M. Efimov ${ }^{1}$, O.S. Afanasenko ${ }^{2}$, \\ E.K. Khlestkina ${ }^{1,3}$ \\ ${ }^{1}$ Institute of Cytology and Genetics SB RAS, Novosibirsk, Russia \\ ${ }^{2}$ All-Russian Research Institute for Plant Protection, St.-Petersburg, Russia \\ ${ }^{3}$ N.I. Vavilov All-Russian Research Institute of Plant Genetic Resources (VIR), St.-Petersburg, Russia \\ *e-mail: bykova@bionet.nsc.ru
}

Key words: Hordeum vulgare, SNP, Cochliobolus sativus, Pyrenophora teres F. teres

Motivation and Aim: Spot blotch, caused by Cochliobolus sativus, and net blotch, caused by Pyrenophora teres F. teres are two of the most widespread and harmful diseases in barley. Identification of genetic loci associated with resistance to both $C$. sativus and $P$. teres $F$. teres is of importance for future marker-assisted selection. The goal of the current study was to identify loci conferring seedling resistance to different pathotypes of $C$. sativus and $P$. teres F. teres in the Siberian spring barley core collection.

Methods and Algorithms: A total of 96 spring barley cultivars and lines were phenotyped at the seedling stage with two C. sativus isolates $(\mathrm{Kr} 2$ and $\mathrm{Ch} 3)$ and four P. teres isolates (S 10.2, K 5.1, P 3.4.0, A 2.6.0). About 16-17 \% and 10-23\% of genotypes were resistant to spot blotch and net blotch isolates, respectively, and 26-30\% and 5-17\% were moderate-resistant to spot and net blotch isolates, respectively. A total of 94 genotypes were analyzed with the barley 50K Illumina Infinium iSELECT assay. From 44,040 SNPs, 40,703 were scorable, from which 39,140 were polymorphic. 27,319 SNPs passed filtering threshold and were used for association mapping. The data were assessed using Microsoft Excel, PASS, Tassel 5.

Results: Data analysis by GLM revealed 3 and 27 SNPs for Kr2 and Ch3 spot blotch isolates, respectively, and 2, 27, 2, 26 SNPs for S10.2, K5.1, P3.4.0, A2.6.0 net blotch isolates, respectively. A total of three genomic regions were associated with the resistance to spot blotch on chromosomes $1 \mathrm{H}, 2 \mathrm{H}, 3 \mathrm{H}$ and three genomic regions were associated with resistance to net blotch on chromosomes $2 \mathrm{H}, 3 \mathrm{H}$ and $6 \mathrm{H}$.

Conclusion: Three genomic regions associated with the resistance to one or both isolates of $C$. sativus and to one of isolates of $P$. teres $F$. teres were identified via screening of the Siberian spring barley core collection. Comparison of their location with QTLs revealed previously either with biparental mapping populations studies or with GWAS of distinct germplasm and other isolates, demonstrated that resistance to isolates of all pathogens is conferred by known spot blotch and net blotch resistance loci. Information on SNPs related can be used further for development of DNA-markers convenient for diagnostics of resistance-associated alleles in barley breeding programs.

Acknowledgements: This study was supported by RSF No. 16-14-00086. 